\title{
Neutron polarimetry in the momentum range 1-6 GeV
}

\author{
Ying Wang $^{1 \star}$, Dominique Marchand ${ }^{1}$, Nikolay Piskunov², and Egle Tomasi-Gustafsson ${ }^{3}$ \\ ${ }^{1}$ Institut de Physique Nucléaire d'Orsay, CNRS/IN2P3 and Univ Paris-Sud, UMR 8608, Univ Paris-Saclay, \\ 91406 Orsay, France \\ ${ }^{2}$ Joint Institute for Nuclear Research, VBLHE, 141980 Dubna, Russia \\ ${ }^{3}$ CEA, DRF, IRFU, SPhN, Saclay, 91191 Gif-sur-Yvette Cedex, France
}

\begin{abstract}
In the framework of the ALPOM II experiment, we compare the figure of Merit of two processes for the neutron analyzing power measurement in the reactions $\vec{n}+p \rightarrow \vec{n}+p$ and $\vec{n}+p \rightarrow p+\vec{n}$ in the beam momentum range from 1 to $6 \mathrm{GeV}$. Based on a pole model calculation for the cross sections and existing data for analyzing powers, this study suggests that for neutron momentum larger than $3 \mathrm{GeV}$, the process of $\vec{n}+p \rightarrow p+\vec{n}$ is more effective for polarimetry.
\end{abstract}

\section{Introduction}

Since Akhiezer and Rekalo suggested the double polarization method for form factors (FFs) measurements in 1968, proton and neutron polarimetries have been revealed to be the most sensitive way to access the nucleon electric to magnetic FFs ratio $[1,2]$. The ratio of the electric to magnetic FFs can be directly measured from the ratio of transverse to longitudinal polarization of the recoil nucleon in the $e N$ scattering induced by linearly polarized electrons.

As the measurement of the longitudinal and transverse polarizations (directly related to GE/GM) are done simultaneously, measurements are affected by less systematic uncertainties. Moreover, as radiative corrections mostly cancel in the polarization ratio (at least the factorized terms), radiative corrections affect much less the results than for the Rosenbluth method, based on unpolarized cross section measurements [3].

At Jefferson Lab, the first double polarization experiment dedicated to measure the proton FF ratio was performed in 1998 at transfer momentum $Q^{2} \simeq 3.5 \mathrm{GeV}^{2}$ benefiting from the technological advances of the Continuous Electron Beam Accelerator Facility (CEBAF) providing a high current polarized beam [4].

Recently, measurements have been extended to $Q^{2}$ around $9 \mathrm{GeV}^{2}$ for the proton electromagnetic FFs ratio [5]. The planned approved experiments are expected to measure the proton FFs ratio up to $Q^{2} 12-15 \mathrm{GeV}^{2}$ [6]. In the neutron case, it is mandatory to use the polarization method for neutron to extract the FFs ratio, due to the smallness of the neutron electric FF. Indeed, before it was possible to apply the polarization method, the data for the neutron FFs ratio could not have the same accuracy as for proton.

\footnotetext{
^e-mail: wangying@ipno.in2p3.fr
} 
The principle of a polarization measurement is the precise determination of the azimuthal asymmetry of the particle. As the asymmetry becomes very small at high momentum, the polarization experiments can be very time consuming. In order to optimize the polarimetry, it is necessary to choose the most suitable reaction. The measurement of proton analyzing powers had been done by ALPOM at beam momentum 1.75 to $5.3 \mathrm{GeV}$ [7]. To study the neutron analyzing power, elastic $n p$ scattering was used as polarizing reaction up to a momentum of $3 \mathrm{GeV}$ [8]. It appears that the neutron analyzing power becomes smaller and smaller at forward angles with increasing energy, whereas it becomes large and negative when the outgoing proton is detected at backward angles [9]. The latter situation corresponds to charge exchange reactions (neutron backward, named CE) and gives a new opportunity to extend neutron polarimetry to high energies.

The purpose of this work is to give an estimation of the expected figure of Merit (FoM) of a polarimeter based on reactions $\vec{n}+p \rightarrow \vec{n}+p$ (neutron forward, named ZE) and $\vec{n}+p \rightarrow p+\vec{n}$ in the ALPOM II energy range with polarized proton and neutron beam momentum up to $7.5 \mathrm{GeV}$ and $4.5 \mathrm{GeV}$ respectively. The present results are based on existing data with the help of the calculation from [10]. The purpose of this paper is to determine at which incident momentum ZE and CE become competitive in terms of FoM.

The experiment ALPOM II is the upgrade of ALPOM [7], which will be carried out at the Veksler and Baldin Laboratory of High Energies (VBLHE) of Joint Institute for Nuclear Research (JINR) in Dubna, produced from the breakup of the deuteron beam accelerated at the Nuclotron. In order to do the measurement at high momentum, a hadron calorimeter has been added to the setup, which makes possible to select particles through their energy deposit.

\section{Performance of the Polarimetry}

The performance of a polarimeter is expressed in terms of the FoM, $\mathcal{F}$. For a given configuration, as target thickness, acceptance..., it is defined as

$$
\mathcal{F}^{2}=\int_{\theta} \varepsilon(\theta) A_{y}^{2}(\theta) d \theta
$$

The integration is performed over the angular range where we assume that the polarimeter is ideally efficient. In (1), $\varepsilon(\theta)$ is the differential efficiency of the process, defined as the differential cross section for the useful reaction (in our case the $\mathrm{CE}$ or $\mathrm{ZE}$ reaction) integrated over the azimuthal angle $\phi$, normalized to the total cross section, $\sigma_{t o t}$, in order to have a relative normalization. And the notation $A_{y}$ is for the analyzing power, the single spin polarization observable when the beam is polarized. The total $n p$ cross section in the energy range of interest is quite constant and it is taken as $\sigma(N+N \rightarrow$ anything $) \simeq 40 \mathrm{mb}$. We can estimate the cross section on a $\mathrm{CH} 2$ target as follows:

$$
\sigma_{\text {tot }}(N+C H 2 \rightarrow \text { anything })=2 \sigma(N+N)+A^{2 / 3} \sigma(N+N) \simeq(80+200) m b=280 m b .
$$

The knowledge of FoM allows to estimate the number of incident events, $N_{\text {inc }}$, necessary to obtain a given error $\Delta P$ on the polarization measurement. It allows to estimate the duration of the experiment. The larger is the FoM, the shorter is the running time to get the same error on the polarization:

$$
N_{\text {inc }}=\frac{2}{\Delta P^{2} \mathcal{F}^{2}} \text {. }
$$

\subsection{Efficiency}

The efficiencies for these two processes are calculated from the cross sections, assuming an ideal system with $100 \%$ experimental efficiency. The formula is, 


$$
\varepsilon(\cos \theta)=\frac{d \sigma / d \cos \theta}{\sigma_{t o t}}
$$

The two diagrams of figure 1 are considered:

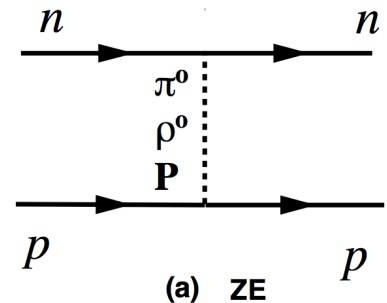

(a) ZE

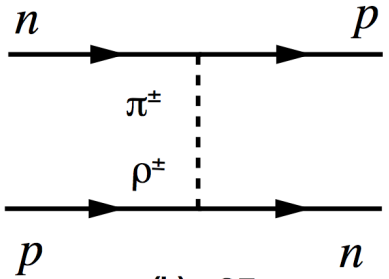

(b) CE

Figure 1. Diagrams of the pole model of elastic $n p \rightarrow n p(p n)$ scattering.

$$
\begin{aligned}
& n\left(k_{1}\right)+p\left(k_{2}\right) \rightarrow n\left(k_{3}\right)+p\left(k_{4}\right) t-\operatorname{channel}(Z E), \\
& n\left(k_{1}\right)+p\left(k_{2}\right) \rightarrow p\left(k_{4}\right)+n\left(k_{3}\right) u-\operatorname{channel}(C E)
\end{aligned}
$$

The Mandelstam variables are defined in terms of four-momenta as:

$$
\begin{aligned}
t & =\left(k_{1}-k_{3}\right)^{2}=\left(k_{2}-k_{4}\right)^{2}, \\
u & =\left(k_{1}-k_{4}\right)^{2}=\left(k_{2}-k_{3}\right)^{2}, \\
s & =\left(k_{1}+k_{2}\right)^{2}=\left(k_{3}+k_{4}\right)^{2} .
\end{aligned}
$$

We use the same mass for neutron and proton $M_{n}=M_{p}=M$.

The formula and parameters of the pole model for $t$-dependent differential cross section are taken from [10],

$$
\frac{d \sigma}{d t}=\frac{1}{64 \pi s q^{2}}\left(\left|T_{\pi}(u)+T_{\rho}(u)\right|^{2}+\frac{1}{4}\left|T_{\pi}(t)+T_{\rho}(t)\right|^{2}+\left|T_{P}(t)\right|^{2}\right)
$$

where $q^{2}=s / 4-M^{2}$ and $T_{\pi / \rho, P}$ are amplitudes corresponding to $\pi, \rho$, pomeron exchanges respectively. The predictions following the (7), are illustrated in figure 2 for different values of the incident neutron beam momentum. The model gives a good description of the data for all values of the beam momentum at small $t$, and a small deviation at large values of $t$. However, the differences are quite small, always within a factor of 10 . We plot in log-scale to highlight the small difference between data and model (figure 2). As expected, ZE is dominant at forward angles, while CE is dominant for backward angles. The same result can also be seen in figure 3 (left) where the $s$-dependence of the cross section is calculated for $t=0$, in the forward region. The total cross sections, integrated over the full angular range, are shown in figure 3 (right). Both show a decreasing behavior when the momentum increases. The difference between $\mathrm{CE}$ and $\mathrm{ZE}$ contributions grows in the region from 0 to $2 \mathrm{GeV}$, and above $2 \mathrm{GeV}$, it becomes constant. According to the calculation of the efficiency, figure 3 shows that the ZE channel dominates the cross section. The other component of FoM formula, the analyzing power, is discussed in the next section. 

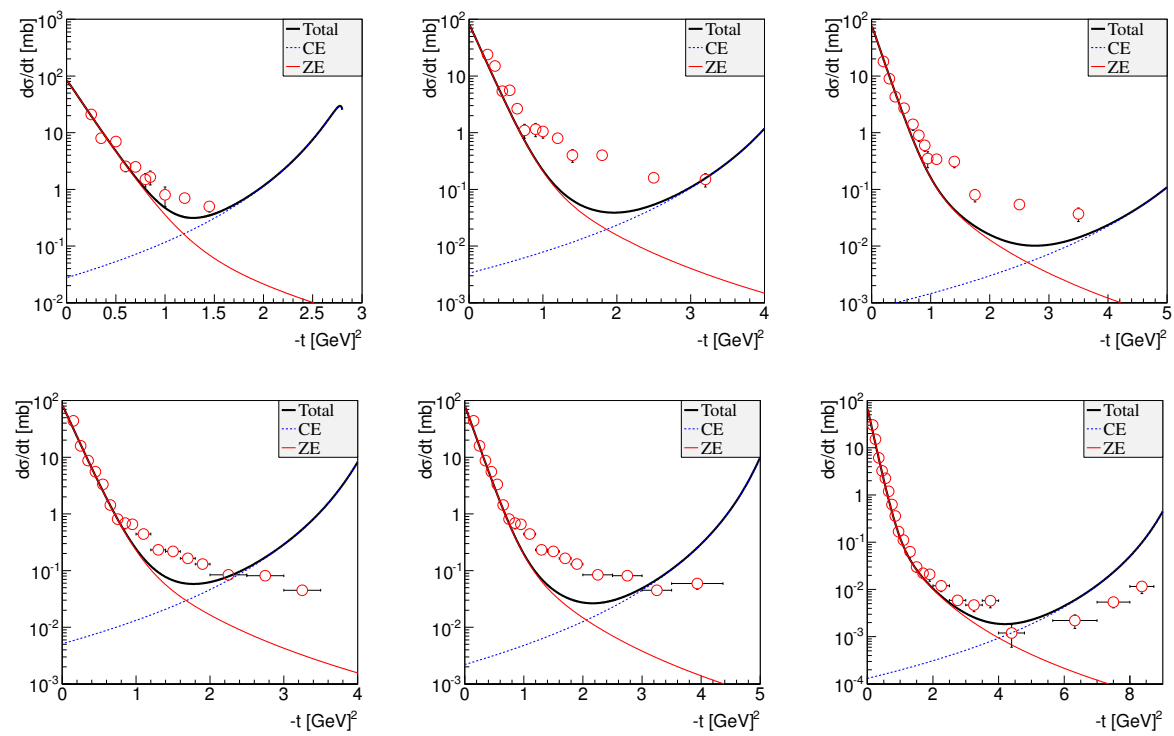

Figure 2. Top figures: data are from [11], corresponding to different incident beam momenta: $P_{\text {Lab }}=2.241 \mathrm{GeV}$ (left), $3.305 \mathrm{GeV}$ (middle), and $4.337 \mathrm{GeV}$ (right). Bottom figures: data are from [12] and correspond to beam momenta: $P_{\text {Lab }}=3.05 \mathrm{GeV}$ (left), $3.570 \mathrm{GeV}$ (middle), $6.120 \mathrm{GeV}$ (right). The model prediction, [10], is also shown (black thick solid line) together with the ZE (red solid line) and CE (blue dotted line) contributions.
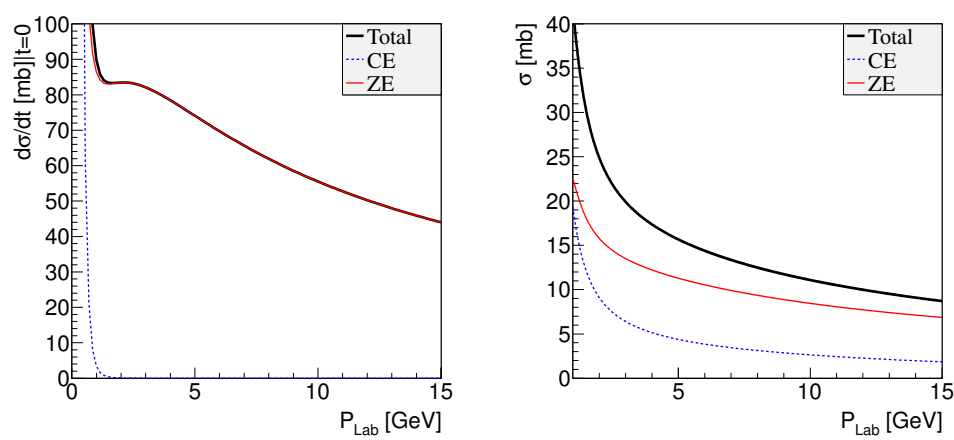

Figure 3. (Left: differential cross section as a function of $P_{\text {Lab }}$, according to (7) for $t=0$. Reference [10], right: total cross section as a function of $P_{L a b}$ (black solid thick line), ZE (thin red solid line), and CE (blue dotted line).

\subsection{Analyzing Powers}

To compare the analyzing powers of $\mathrm{CE}$ and ZE reactions, in figure 4 we plot the maximum absolute value of the polarization as a function of the incident momentum, with a linear function to extrapolate to the higher momentum region. The results have to be considered upper limits. With increasing momentum, the maximum absolute values of $\mathrm{ZE}$ and $\mathrm{CE}$ have an opposite behavior. While the ZE analyzing power decreases with increasing momentum, the $\mathrm{CE}$ analyzing power increases, which compensates the cross section contribution of CE reaction. 


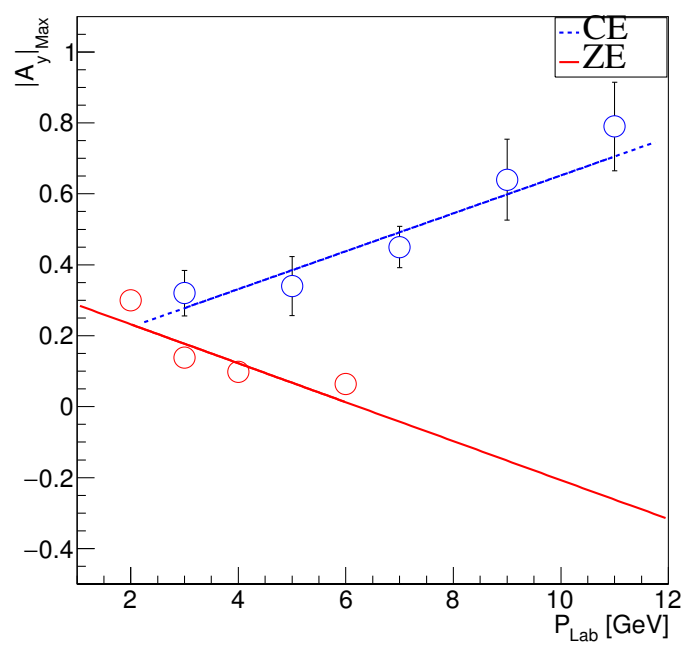

Figure 4. $\left|\mathrm{A}_{y}\right|_{\text {Max }}$ for $n p \rightarrow n p(p n)$ based on the polarization data from CE (blue dotted line) from [9] and ZE (red solid line) from [13] with the parameters as in table 1

Table 1. ZE parameters corresponding to figure 4.

\begin{tabular}{|c|c|}
\hline \hline CE & $\mathrm{y}=0.1765+0.0425 \mathrm{x}$ \\
\hline $\mathrm{ZE}$ & $\mathrm{y}=0.35-0.0536 \mathrm{x}$ \\
\hline \hline
\end{tabular}

\subsection{Figure of Merit}

The squared differential FoM (for CE and ZE) is defined as:

$$
\mathcal{F}^{2}(\cos \theta)=A_{y_{(\operatorname{Max})}}^{2} \cdot \varepsilon(\cos \theta)
$$

Following this definition we calculate the integrated FoM (see figure 5) in the full angular range. As discussed above, the cross sections of both reactions decrease with increasing momentum, but CE is far smaller than ZE in the low momentum region, where the analyzing powers do not compensate this effect. So, in this momentum region, ZE has larger FoM. But for larger values of $p_{\text {Lab }}$, above $3 \mathrm{GeV}$, the analyzing power having a more important weight than the cross section in the figure of Merit, the CE reaction gives larger FoM, which is more effective for polarimetry. For the same running time, the measurement will be more precise, having smaller errors. In figure 5 the red empty circles are calculated from the Monte Carlo simulation of JLab proposal PR-11-009 on the basis of the ZE reaction [14]. This comparison is done for an ideal apparatus with $100 \%$ efficiency and acceptance. The interesting momentum range has to be validated by experimental data. The crossing point, attributing a factor of ten uncertainty in the cross section, may vary in the range 3 to $5 \mathrm{GeV}$.

\section{Discussion and conclusion}

In ALPOM II, at momenta of $3 \mathrm{GeV}$ and higher, assuming $10^{6}$ incident particles, the uncertainty of the polarization $(\triangle \mathrm{P})$ can be estimated to $0.045(\mathrm{ZE})$ and $0.030(\mathrm{CE})$, in spite of the fact that 


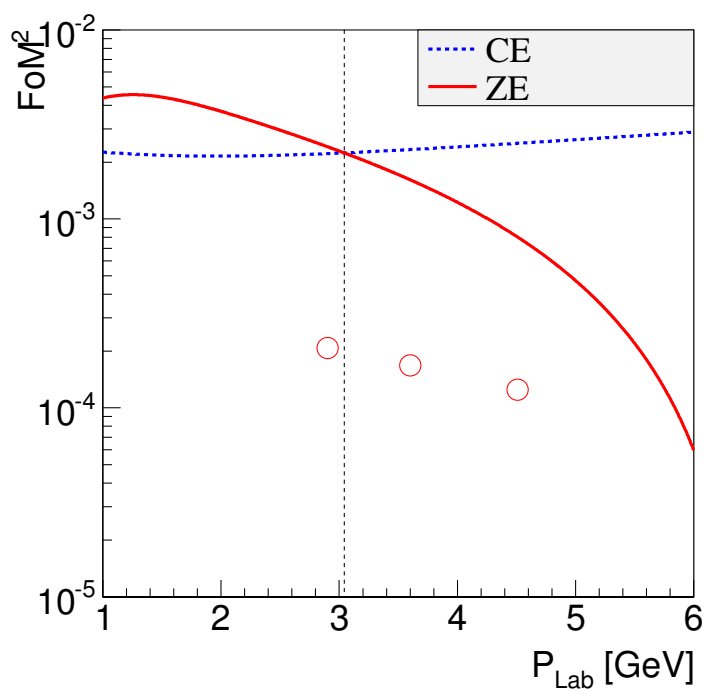

Figure 5. Integrated figure of Merit for $n p \rightarrow n p(p n)$ based on the fitting result from figure 4 (upper limit) for CE (blue dotted line) and ZE (red solid line). The red empty circles are calculated from the Monte Carlo simulation of JLab proposal PR-11-009.

the total cross section for ZE is larger than for CE. With increasing beam momentum, FoM of ZE decreases dramatically while CE stays about constant, then the CE becomes preferable, under the present assumptions of ideal experimental environment. However, our calculation can predict elastic scattering only up to $6 \mathrm{GeV}$, where analyzing powers have been measured. The model is not reliable for further extrapolation. Moreover, the uncertainty on the calculated figure of merit is essentially related to the uncertainty of the calculation and the lack of experimental data. Attributing a factor of 10 uncertainty on the $\mathrm{CE}$ cross section, affects the figure of merit (squared) by the same factor. The crossing is expected in a range of $\Delta P$ of 3 to $5 \mathrm{GeV}$.

In conclusion, we have shown the results of the comparison for FoMs of $\mathrm{CE}$ and $\mathrm{ZE}$ processes and we suggest to use the $\mathrm{CE}$ reaction for the neutron analyzing power measurement when the beam momentum is above $3 \mathrm{GeV}$. In ALPOM II experiment, the proton analyzing power will also be measured which is useful for the proton FF measurement [15].

\section{References}

[1] A. Akhiezer, M.P. Rekalo, Sov.Phys.Dokl. 13, 572 (1968)

[2] A. Akhiezer, M.P. Rekalo, Sov.J.Part.Nucl. 4, 277 (1974)

[3] M. Rosenbluth, Phys.Rev. 79, 615 (1950)

[4] H. Anklin, L. deBever, K. Blomqvist, W. Boeglin, R. Böhm, M. Distler, R. Edelhoff, J. Friedrich, D. Fritschi, R. Geiges et al., Physics Letters B 428, 248 (1998)

[5] A. Puckett, E. Brash, O. Gayou, M. Jones, L. Pentchev et al., Phys.Rev. C 85, 045203 (2012). 1102.5737

[6] L.P. Pentchev, C.F. Perdrisat, C. Cisbani, V. Punjabi, B. Wojtsekhowski, JLab E12-07-109 (2007) 
[7] L. Azhgirey, V. Arefev, S. Basilev, Y. Bushuev, V. Glagolev et al., Nucl.Instrum.Methods (2004)

[8] T. Eden, a. Madey, R. et, Phys. Rev. C 50, R1749 (1994)

[9] M.A. Abolins, M.T. Lin, R.C. Ruchti, J.G. Horowitz, R.C. Kammerud, N.W. Reay, K. Reibel, N.R. Stanton, K.W. Edwards, D.G. Crabb et al., Phys. Rev. Lett. 30, 1183 (1973)

[10] Y. Troyan, M. Anikina, A. Belyaev, A. Ierusalimov, A. Troyan, Physics of Particles and Nuclei Letters 11, 101 (2014)

[11] M.N. Kreisler, F. Martin, M.L. Perl, M.J. Longo, S.T. Powell, Phys. Rev. Lett. 16, 1217 (1966)

[12] B.G. Gibbard, L.W. Jones, M.J. Longo, J.R. O’Fallon, J. Cox, M.L. Perl, W.T. Toner, M.N. Kreisler, Phys. Rev. Lett. 24, 22 (1970)

[13] R. Diebold, D.S. Ayres, S.L. Kramer, A.J. Pawlicki, A.B. Wicklund, Phys. Rev. Lett. 35, 632 (1975)

[14] J. Arrington et al., JLab PR-11-009 (2011)

[15] N. Piskunov et al., np charge exchange polarimetry in GeV region. Report at XXIII International Baldin Seminar on High Energy Physics "Problems, Relativistic Nuclear Physics and Quantum Chromodynamics" (Dubna, Russia, September 19-24, 2016).

http://relnp.jinr.ru/ishepp-xxiii/Presentations/Piskunov.pdf 